Wright State University

CORE Scholar

Physics Faculty Publications

Physics

4-1997

\title{
A Fast Scan Submillimeter Spectroscopic Technique
}

Douglas T. Petkie

Wright State University - Main Campus, dpetkie@yahoo.com

Thomas M. Goyette

Ryan P. A. Bettens

Sergei P. Belov

Sieghard Albert

See next page for additional authors

Follow this and additional works at: https://corescholar.libraries.wright.edu/physics

Part of the Physics Commons

\section{Repository Citation}

Petkie, D. T., Goyette, T. M., Bettens, R. P., Belov, S. P., Albert, S., Helminger, P., \& De Lucia, F. C. (1997). A Fast Scan Submillimeter Spectroscopic Technique. Review of Scientific Instruments, 68 (4), 1675-1683. https://corescholar.libraries.wright.edu/physics/786

This Article is brought to you for free and open access by the Physics at CORE Scholar. It has been accepted for inclusion in Physics Faculty Publications by an authorized administrator of CORE Scholar. For more information, please contact library-corescholar@wright.edu. 


\section{Authors}

Douglas T. Petkie, Thomas M. Goyette, Ryan P. A. Bettens, Sergei P. Belov, Sieghard Albert, Paul Helminger, and Frank C. De Lucia 


\title{
A fast scan submillimeter spectroscopic technique
}

Douglas T. Petkie, Thomas M. Goyette, Ryan P. A. Bettens, S. P. Belov, ${ }^{\text {a) }}$

Sieghard Albert, Paul Helminger, ${ }^{\text {b) }}$ and Frank C. De Lucia

Department of Physics, The Ohio State University, Columbus, Ohio 43210

(Received 19 September 1996; accepted for publication 25 November 1996)

\begin{abstract}
A new fast scan submillimeter spectroscopic technique (FASSST) has been developed which uses a voltage tunable backward wave oscillator (BWO) as a primary source of radiation, but which uses fast scan $\left(\sim 10^{5}\right.$ Doppler limited resolution elements/s) and optical calibration methods rather than the more traditional phase or frequency lock techniques. Among its attributes are (1) absolute frequency calibration to $\sim 1 / 10$ of a Doppler limited gaseous absorption linewidth $\left(<0.1 \mathrm{MHz}, 0.000003 \mathrm{~cm}^{-1}\right)$, (2) high sensitivity, and (3) the ability to measure many thousands of lines/s. Key elements which make this system possible include the excellent short term spectral purity of the broadly $(\sim 100 \mathrm{GHz})$ tunable BWO; a very low noise, rapidly scannable high voltage power supply; fast data acquisition; and software capable of automated calibration and spectral line measurement. In addition to the unique spectroscopic power of the FASSST system, its implementation is simple enough that it has the prospect of impacting a wide range of scientific problems. (C) 1997 American Institute of Physics. [S0034-6748(97)02503-3]
\end{abstract}

\section{INTRODUCTION}

\section{A. Applications and impact of millimeter and submillimeter spectroscopy}

High resolution millimeter and submillimeter $(\mathrm{mm} /$ submm) spectroscopy has had a major impact on many important fields of science and technology. The earliest studies in this region were of small, fundamental species such as $\mathrm{H}_{2} \mathrm{O}, \mathrm{O}_{2}, \mathrm{NO}, \mathrm{CH}_{3} \mathrm{~F}$, and OCS and served to both establish spectroscopic methodologies and to provide basic information about molecular structure and interactions. ${ }^{1-3} \mathrm{Be}-$ cause these small, fundamental species have intrinsically interesting collisional properties, their dynamical properties have been studied as well. These studies have ranged from investigations of pressure broadening near room temperature (which are fundamental to the deconvolution of atmospheric remote sensing data) $)^{4-6}$ to basic studies of the quantum nature of molecular collisions at low temperature. ${ }^{7,8}$

Because the strength of the interaction between electromagnetic radiation and molecular rotation peaks sharply in the $\mathrm{mm} / \mathrm{sub} \mathrm{mm}$ region, a variety of spectroscopically based remote sensing applications have grown out of this more basic work. The least remote of these have involved laboratory studies of molecular lasers and the collision induced rotational and vibrational processes which are central to their operation. ${ }^{9-11}$ This spectral region has also played an important role in the study of the chemical processes in the upper atmosphere which are important in ozone formation and destruction. ${ }^{12-14}$ Finally, the vast majority of the over 100 molecular species which have been identified and studied in the interstellar medium have been observed by means of $\mathrm{mm} / \mathrm{sub} \mathrm{mm}$ "radio", astronomy. ${ }^{15-18}$

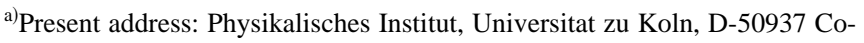
logne, Germany.

${ }^{b}$ Present address: Department of Physics, University of South Alabama, Mobile, AL 36688.
}

\section{B. Current spectroscopic practice: The source and detector problem}

In spite of these and other applications, the $\mathrm{mm} / \mathrm{sub} \mathrm{mm}$ spectral region is by far the least explored portion of the electromagnetic spectrum, largely because of the difficulty of generating and detecting radiation at these wavelengths. However, over the years, a number of approaches have been developed. High resolution spectroscopy was first extended into the submillimeter spectral region by means of nonlinear harmonic generation ${ }^{3}$ and the technique extended by the introduction of sensitive helium temperature detectors ${ }^{19}$ and improved harmonic generator design. ${ }^{20}$ Nonlinear techniques have also been used to generate difference frequencies between optical lasers ${ }^{21-23}$ and to produce microwave sidebands on far infrared (FIR) laser sources. ${ }^{24,25}$ Additionally, a number of solid state diode sources have been extended to higher frequency. ${ }^{26,27}$

Of all of the techniques, those most closely related to the fast scan submillimeter spectroscopic technique (FASSST) system described here are also based on backward wave oscillator (BWO) tubes. In 1953, Kompfner and Williams demonstrated that self-sustained oscillation in traveling wave structures could be achieved by the interaction between the oppositely directed group and phase velocities. ${ }^{28}$ Chief among the attributes of these devices is the broad band electrical tunability which results from the dispersion relation of this backward wave interaction. Among the most successful implementations of this concept were the carcinotrons of Tompson $\mathrm{CSF}^{29}$ and the BWOs of the ISTOK Research and Development Company of Fryazino, Moscow Region, Russia. $^{30,31}$

The latter are particularly advantageous for spectroscopy because of their broad bandwidth, good spectral purity, high frequency capability, and relatively small power consumption. The ISTOK BWOs have been used successfully in freerunning spectrometers, ${ }^{32,33}$ and in synthesized phase-locked systems. ${ }^{34-36}$ A large amount of important submillimeter 


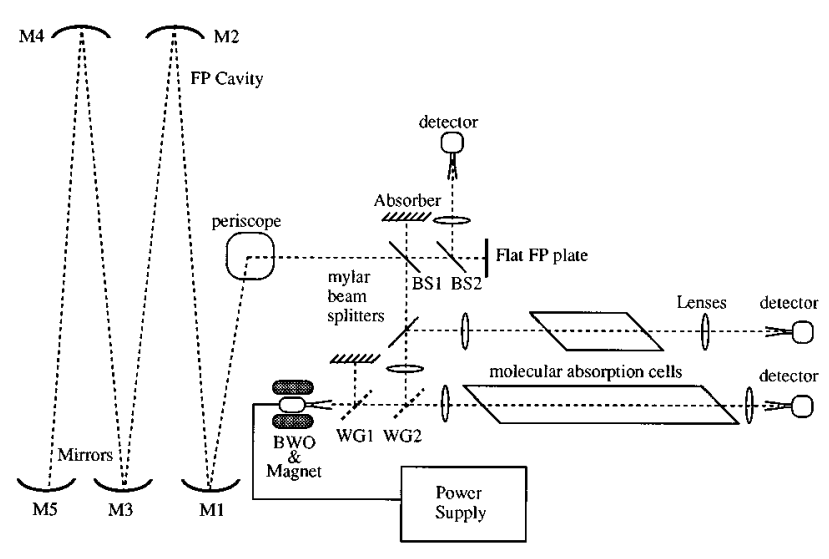

FIG. 1. Block diagram of the FASSST system.

spectroscopy has been accomplished with these systems, and interested readers are referred to the cited references for details.

\section{A new spectroscopic system for the $\mathrm{mm} / \mathrm{sub} \mathrm{mm}$ spectral region}

This article describes a new high resolution spectroscopic system for the $\mathrm{mm} / \mathrm{sub} \mathrm{mm}$ spectral region, which is fast, broadband, sensitive, and simple. It is based on broadband, voltage tunable BWOs produced by the ISTOK Research and Development Company of Fryazino, Moscow Region, Russia and uses very fast sweep $\left(\sim 10^{5}\right.$ Doppler limited spectral resolution elements per second) and optical calibration methods to replace the phase and frequency lock techniques more commonly used.

Because the fast scan effectively "freezes" frequency instability on the time scale of the optical calibration period, resolution, and frequency measurement accuracy are comparable to that of the much slower and more complex phase/ frequency lock systems. This FASSST approach makes it possible to fully utilize the inherent instantaneous bandwidth of the BWOs and to achieve a spectroscopic system of unprecedented capabilities. Furthermore, the resultant system is much simpler and has the potential for wide application.

\section{SYSTEM OVERVIEW}

\section{A. The basic spectroscopic system}

Figure 1 shows a block diagram of the FASSST system. In this system, an ISTOK OB-30 is used to cover the 240$375 \mathrm{GHz}$ region. Similar tubes are available from ISTOK for the $\sim 100-1000 \mathrm{GHz}$ region. The first wire grid polarizer (WG1) provides a well defined polarization from the output of the overmoded BWO waveguide. The second polarizer (WG2) is used to split the output power of the BWO, with $\sim 90 \%$ being directed quasioptically through the molecular absorption cell and detected by an InSb hot electron bolometer operating at $1.5 \mathrm{~K}$. The remaining $\sim 10 \%$ of the power is coupled into a Fabry-Perot (FP) cavity via a mylar beamsplitter (BS1), which provides fringes for frequency interpolation between reference spectral lines of known frequency. In order to provide a highly accurate basis for the analysis of the frequency-voltage characteristic of the BWO, a folded FP cavity of length $\sim 38.89 \mathrm{~m}$ is used to provide modes every $\sim 3.854 \mathrm{MHz}$. The use of more compact FP cavities in subsequent versions of this system will be discussed below. Provision for a second molecular absorption cell which can be used for calibration purposes is also provided.

\section{B. The key system elements}

The key system elements include:

(1) The most fundamental element is the excellent short term spectral purity of the BWO. From studies over many years, it has been observed that the short-term spectral purity of free-running ISTOK BWOs is $\sim 10 \mathrm{kHz}^{37}$ Without this spectral purity, the FASSST system would not be possible.

(2) Second, the BWOs can be voltage tuned continuously over an $\sim 50 \%$ frequency range, which contains $\sim 10^{5}$ spectral resolution elements (Doppler limited).

(3) The synthesized frequency reference system typical of high resolution submillimeter spectrometers is replaced by a system more typical of optical spectroscopy. However, the longer wavelength significantly relaxes the requirements for optical precision and much greater frequency accuracy can be achieved.

(4) A fast $\left(\sim 10^{5}\right.$ spectral resolution elements/s, currently limited by detector bandwidth) sweep and data acquisition system freeze any drift in the source frequency over the time required to sweep from one reference fringe to the next. This eliminates the need for active frequency stabilization.

(5) Fast data acquisition and calibration hardware and software. In a very general sense, the bandwidth of this system plays the same role as the bandwidth of the lock loops of more traditional systems.

\section{System attributes}

The combination of these five elements make it possible to measure thousands of spectral lines per second, with a frequency accuracy of a small fraction of a Doppler width $\left(\sim 0.1 \mathrm{MHz} / 3 \times 10^{-6} \mathrm{~cm}^{-1}\right)$. Signal averaging is straightforward, and for equivalent integration times the sensitivity is the same as for slow-sweep, synthesized phase locked systems. Finally, the system is very simple in both concept and execution and holds the promise of being used in a wide variety of applications.

\section{EXPERIMENTAL DETAILS}

\section{A. Tube characteristics}

The BWO is an electron beam device whose frequency depends on the interactions among the electrons, the periodicity of the BWOs slow wave structure, and the electromagnetic radiation propagating along with the electron beam in the slow wave structure. These are often described in the context of backward wave space harmonics and dispersion relations. ${ }^{38}$ Because the electron velocity has a first order effect in these relations, the frequency of oscillation $f$ is strongly dependent on the electric potential $V$ between the 


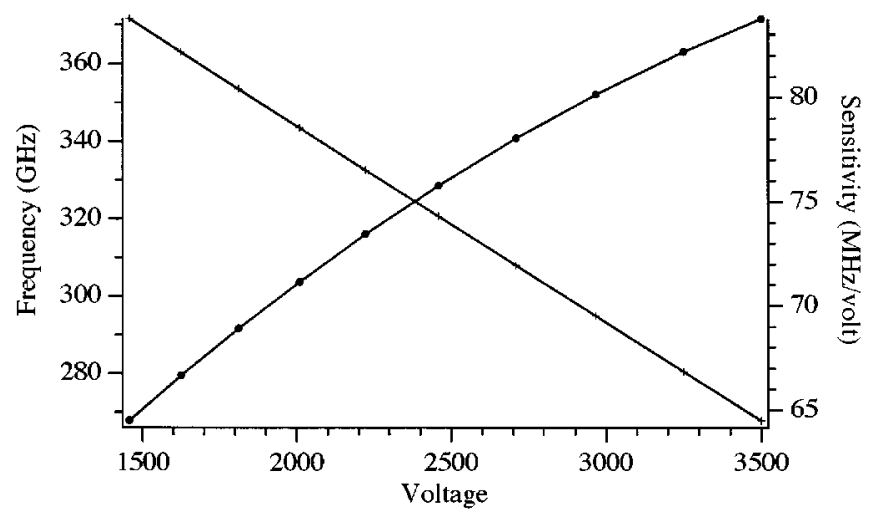

FIG. 2. Dependence of frequency on voltage (left scale, points) and voltage sensitivity (right scale, crosses) for an OB-30 BWO.

cathode and slow wave structure. For an OB-30, $d f / d V \sim 75 \mathrm{MHz} / \mathrm{V}$. This increases somewhat with frequency and for tubes centered around $1 \mathrm{THz}$ is of the order $\sim 100 \mathrm{MHz} / \mathrm{V}$. For a given tube, $d f / d V$ typically decreases with increasing voltage, largely because of the quadratic relation between electron energy and velocity. Figure 2 shows the functional dependence of frequency as well as $d f / d V$ on voltage for the OB-30 used in this work.

Because the slow wave structure does not have a resonant frequency, BWOs can be very broadbanded. However, because of this low $Q$, feedback in the form of power reflected back into the slow wave structure can dramatically alter the frequency and power output of the BWO. In a broadband system in which fast, broad sweeps are executed, it is not possible to control these reflections by narrow band matching techniques, and it is important that the reflections be carefully controlled and minimized. In fact, spontaneous locking to the FP etalon used as a frequency reference is not uncommon unless steps are taken to significantly reduce feedback.

The thermal drift of the frequency of the BWO can be $\sim 500 \mathrm{MHz} / \mathrm{K}$ due to the thermal expansion of the slow wave structure. However, because the time constant for this drift is of the order of a few seconds (a time long in comparison to the interval between FP modes in the fast scan), it is not necessary to thermally stabilize the tube. This is fortunate because the power input to the BWO varies by more than a factor of 2 during the voltage sweep and external thermal control would be of little utility.

Finally, vibrations which change the orientation of the magnetic field which guides the electron beam relative to the slow wave structure of the BWO can modulate both the frequency and power output. In our experience, such vibrations can be reduced below their observational threshold by straightforward techniques in an ordinary laboratory environment.

\section{B. The frequency calibration scheme}

If the frequency of the BWO as a function of voltage were linear, the frequency calibration scheme could be as simple as having two spectral lines of known frequency somewhere near the opposite ends of the frequency sweep range and using linear interpolation. However, from Fig. 3, it

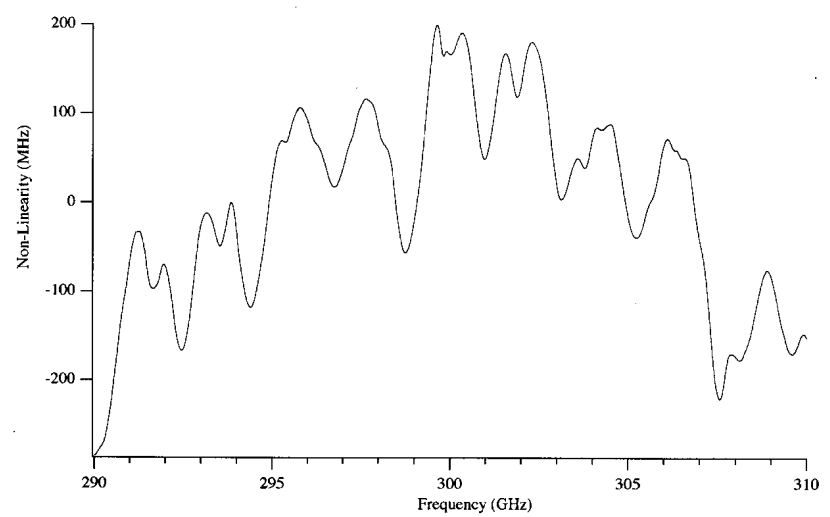

FIG. 3. Typical small scale structure of the frequency-voltage characteristic of a BWO.

is immediately obvious that the small scale structure is much more complex than given by a simple dispersion relation. More specifically, if the effects of the small scale structure are not properly treated, the frequency accuracy of the FASSST system will be $\sim 100 \mathrm{MHz}$, approximately 1000 times worse than that required for high resolution spectroscopy. However, we will demonstrate below that with a small FP mode spacing we have been able to use simple linear interpolation methods to measure line frequencies to $\sim 1 / 10$ of the Doppler limited linewidth.

Based on these considerations, the basic FASSST scheme is to:

(1) take a fast $\left(10^{4}-10^{5} \mathrm{MHz} / \mathrm{s}\right)$ scan over the spectral region of interest,

(2) include two or more (typically $\sim 50$ are available) reference lines,

(3) use the known frequencies of the reference lines to determine the FP cavity mode spacing and absolute frequency,

(4) count FP modes to establish the frequency of each fringe, and

(5) use linear interpolation between the two nearest FP modes to calculate the frequencies of the unknown lines.

Because the thermal history of the BWO effects the frequency-voltage function at this level of precision, each sweep is calibrated separately.

\section{The power supply scheme}

Ultimately the combination of high spectral purity and voltage tunability of these BWOs makes the system described in this article possible. However, these qualities can only be exploited in the context of a power supply with complimentary properties, a stringent requirement. More specifically, the 240-375 GHz OB-30 BWO tunes $\sim 100$ $\mathrm{GHz}$ with a voltage variation of $\sim 1000-3500 \mathrm{~V}$, a tuning rate of $\sim 75 \mathrm{MHz} / \mathrm{V}$. If it is desired that the voltage fluctuations of the power supply result in a frequency variation which is no more than $10 \%$ of a linewidth $(\sim 0.1 \mathrm{MHz})$, they must be less than $\sim 2 \mathrm{mV}$ in the context of a power supply capable of sweeping several thousand volts in $\sim 1 \mathrm{~ms}$. 


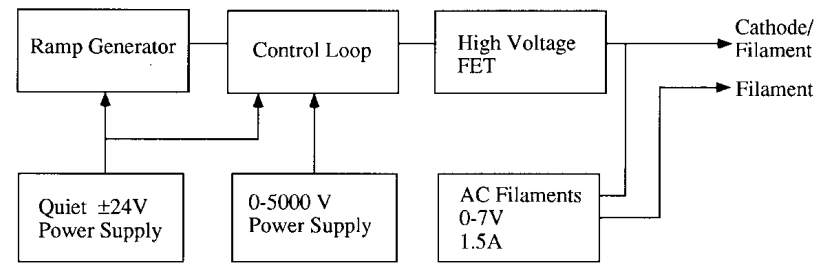

FIG. 4. Block diagram of the power supply sweeper.

The block diagram of the power supply is shown in Fig. 4. Because most of the detailed circuitry is of conventional, albeit careful design, we will not discuss it in detail here. ${ }^{39}$ However, it is important to note that the details of the layout and the resultant capacitive, inductive, and thermal effects are very important. As a result, it is useful to include monitoring circuitry, which allows the observation of very low frequency $(\sim 0.1-1.0 \mathrm{~Hz})$, low amplitude $(<10 \mathrm{mV})$ "rumble" as well as the more conventional ripple and power supply switching effects.

\section{The stabilization scheme}

For the optical calibration scheme to be accurate, the BWO frequency drift must be linear over the time required to scan from one FP cavity mode to the next. In addition to thermal drift, power supply ripple can contribute to a nonlinear sweep between FP cavity modes. In addition to fundamental power supply variations, voltages induced onto the beam accelerating voltage either from the ac filaments of the BWOs or system ground loops will modulate the frequency of the system.

\section{E. Resultant scan strategies}

In our system, the upper limit on the scan speed is set by the $\sim 1 \mathrm{MHz}$ bandwidth of the InSb detector. If the entire $100 \mathrm{GHz}$ of the tube is to be scanned, Doppler widths are $\sim 1 \mathrm{MHz}$, and 10 digitization points per spectral resolution width are required, the minimum sweep time set by the detector bandwidth is $1 \mathrm{~s}$. This is a relatively long time, and the bandwidths required in the power supply sweeper would be low and reasonably straightforward to obtain. However, in many applications only a small portion of the $100 \mathrm{GHz}$ avail-

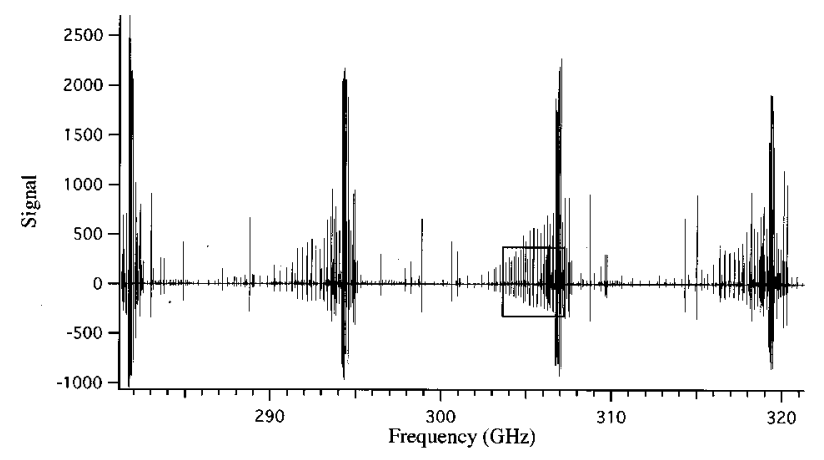

FIG. $5.3 \mathrm{~s}$ scan of $40 \mathrm{GHz}$ of the $\mathrm{mm} / \mathrm{sub} \mathrm{mm}$ spectrum of $\mathrm{HNO}_{3}$ showing four bandheads. The signal amplitude is adjusted to fill the 4048 (12 bit) steps of the A/D converter.

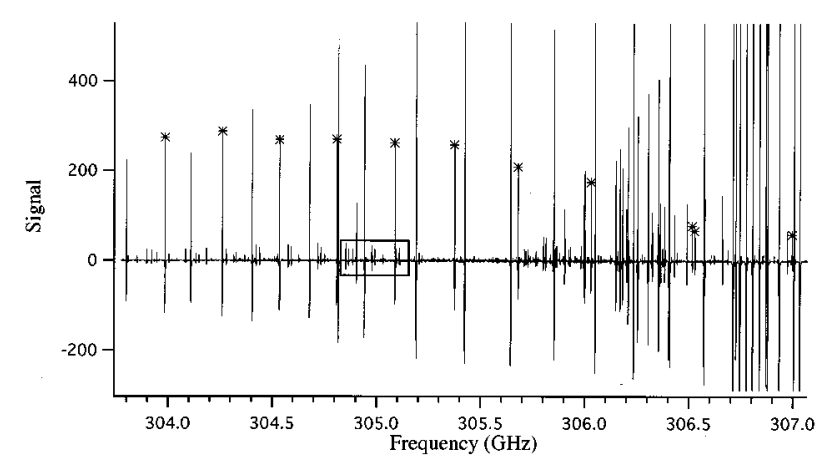

FIG. 6. A graphical blowup of the region shown in the box of Fig. 5. The spectral lines marked with the star belong the $\nu_{7}$ vibrational state.

able will be scanned, but the same sweep rate in terms of FP cavity modes/s is required in order to eliminate drift and ripple effects. As an example, if 20 FP cavity modes $(\sim 100 \mathrm{MHz})$ are scanned rather than the 20000 in the full $100 \mathrm{GHz}$, the $1 \mathrm{~s}$ sweep period can be reduced to $1 / 1000$ of a second.

In both of these examples, the FP marker rate is $20000 /$ s. If the ac ripple effects are of frequency $\sim 100 \mathrm{~Hz}$ and magnitude $V_{\text {ac }}$ their maximum (depending on phase) contribution to the voltage change between FP markers will be reduced from $V_{\mathrm{ac}}$ by the ratio of marker rate of the ripple rate (a factor of 200 in these examples). Since only the nonlinearity of the ripple (which is small over 1/200 of a cycle) contribute to calibration error, these fast scans effectively freeze ripple effects. However, because the contribution of these effects to calibration error will grow as the inverse square of the scan rate, at slower scanning rates, they can become significant.

\section{DIAGNOSTIC RESULTS AND OPERATIONAL PRACTICE}

\section{A. The BWO frequency-voltage characteristic}

As discussed above, both the large and small scale structure of the frequency-voltage function and the time scales of its variation determine the details of the design. In order to

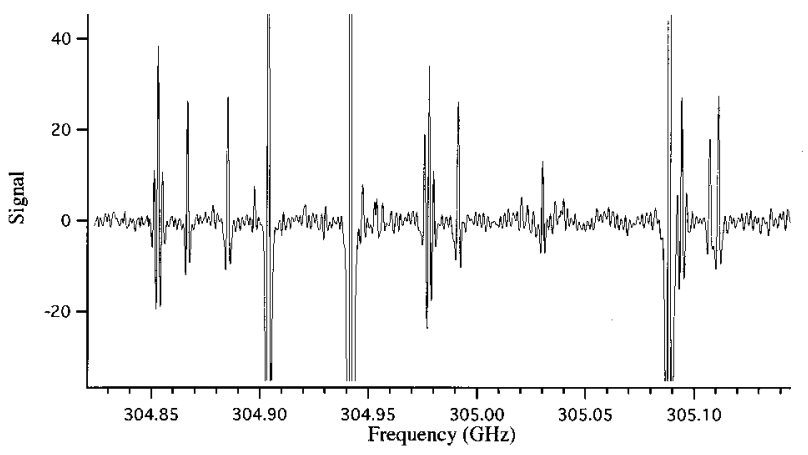

FIG. 7. A graphical blowup of the region shown in the box in Fig. 6. The spectrum has been convolved with a first derivative line shape, resulting in line shapes which are approximately second derivative. The "noise" in the figure results from the digital noise of the A/D converter and is not a fundamental limit on the detection of weak lines. 


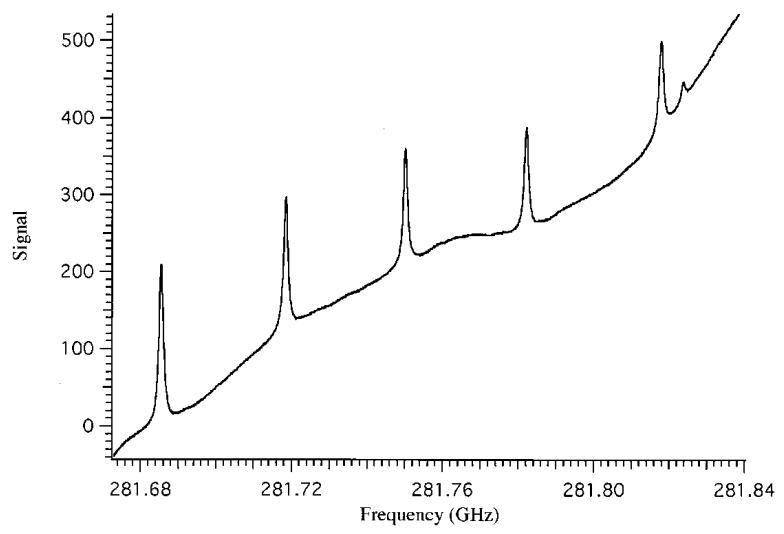

FIG. 8. Portion of a true line shape spectrum recorded for use in linewidth measurements.

determine these structures, the FP fringe spacing as a function of voltage was recorded. This provided the data which resulted in Figs. 2 (the large scale structure) and 3 (the small scale structure). The smooth curve of Fig. 2 closely approximates the basic theory, ${ }^{38}$ whereas Fig. 3 shows deviation from this ideality, primarily caused by reflections, both internal and external to the BWO itself.

\section{B. Typical spectra}

Because a single scan contains $\sim 10^{6}$ resolution elements, it is not possible to graphically display a complete, full band spectrum. However, Figs. 5-7 show a series of blow ups in both frequency and sensitivity of the $\mathrm{mm} / \mathrm{submm}$ spectrum of nitric acid $\left(\mathrm{HNO}_{3}\right)$ which provide a good perspective. In order to maximize the dynamic range of the signal in this spectrum, the low frequency roll off in the analog amplifiers between the detector and the A/D input to the computer was adjusted to provide an approximately first derivative line shape and to generally suppress the recording of the power variations of the BWO. For the study of weaker lines, additional analog gain is used at the expense of the measurement of the amplitude of the stronger lines. In the current system (which is not optimized for sensitivity), strongly absorbing lines are of $\mathrm{S} / \mathrm{N}=\left(10^{4}-10^{5}\right) / 1$ in $1 \mathrm{MHz}$ of bandwidth. Comparison of the closely spaced triplets in Fig. 7 (which are due to the torsional motion in the $\nu_{9}$ excited state) with observations of phase locked systems shows that the spectral linewidths recorded by the FASSST system are also Doppler limited.

If the measurement of linewidths and line shapes are required, all bandwidths in the system are adjusted to preserve "true line shape." A portion of such a spectrum is shown in Fig. 8.

\section{Software details and operational practice}

In order to efficiently utilize the FASSST system's ability to rapidly acquire and store spectral information, it is necessary to develop appropriate software and operational practices. It is useful to first explore the information content of the spectra produced by the FASSST system. In $\sim 1 \mathrm{~s}$,
$10^{6}$ data points which characterize $10^{5}$ resolution spectral elements can be acquired with a dynamic range of $\sim 10^{4}$. This is $\sim 10 \mathrm{MBytes} / \mathrm{s}$ of relatively uncorrelated data. Furthermore, because of the complexity and density of rotational spectra, many of these resolution elements may be filled with observable, resolvable absorptions. Although averaging multiple sweeps to increase $\mathrm{S} / \mathrm{N}$ is possible, the inherent source brightness, detector sensitivity, and strength of the molecular interaction render this unnecessary in many applications. In these circumstances, real system efficiency is then limited by overhead: the cycling of samples through the system and more importantly by the time required to turn raw spectral information into useful scientific results.

In practice the operational procedures are:

(1) The sample cell is filled to approximately 10 mTorr with the gas of spectroscopic interest.

(2) The power supply initiates a sweep and triggers the computer to begin data acquisition. Typically four channels are recorded: the output of the $\mathrm{mm} / \mathrm{submm}$ detector in the absorption cell path, the output of the $\mathrm{mm} / \mathrm{submm}$ detector of the FP cavity, the output voltage sweep, and the trigger. A full tube sweep $(100 \mathrm{GHz})$ takes $1-10 \mathrm{~s}$ and small spectral regions proportionately less time.

(3) If higher signal to noise is desired, additional sweeps are recorded. Since each sweep can be individually calibrated, no assumptions about tube or FP stability are required. Because $\mathrm{mm} / \mathrm{submm}$ spectra typically contain base line interference patterns in addition to the spectral information, provision is made for rapid gas fill/gas pump out cycles so that base line subtraction is possible.

(4) The spectral and FP data file is then scanned automatically for peaks and fitting procedures used to locate (in bin or time space) each peak.

(5) For frequency calibration of the FP modes, the known frequency-voltage characteristic of the BWO tube is used to provide a first approximation so that Fourier transform correlation techniques between an observed reference spectrum and a spectrum calculated from the known spectral parameters of the reference gas can be carried out. In principle, only two previously measured lines per calibration segment (which might vary between 1 and $100 \mathrm{GHz}$ in width) are required to determine the fringe spacing and absolute frequency. However, in practice, many known lines are used in a least squares procedure to determine these two parameters. While in principle, the reference gas might be one selected especially for calibration purposes and contained in the separate absorption cell, in practice many gases have enough measured spectral lines to serve as their own self-reference.

(6) Linear interpolation is then used between each of the FP fringes to establish the frequency of each bin in the data. By comparison of the peak locations (which can be fractional bins) from step (4), the frequency of each unknown line is calculated.

(7) Because the absorption coefficients of spectral lines in the $\mathrm{mm} / \mathrm{sub} \mathrm{mm}$ can be calculated with good accuracy (often better than 1\%), the known absorption coefficients of the lines of the reference gas are used to calibrate the absorption coefficients of the unknown lines, with linear interpola- 
TABLE I. Example of the line list for a portion of the spectrum shown in Fig. 5.

\begin{tabular}{|c|c|c|c|c|c|c|c|c|c|}
\hline$J^{\prime}$ & $K_{a}^{\prime}$ & $K_{c}^{\prime}$ & $J^{\prime \prime}$ & $K_{a}^{\prime \prime}$ & $K_{c}^{\prime \prime}$ & $\begin{array}{l}\text { Vib } \\
\text { state }\end{array}$ & $\begin{array}{l}\text { Absorption } \\
\left(\mathrm{cm}^{-1}\right)\end{array}$ & $\begin{array}{l}\text { Frequency } \\
(\mathrm{MHz})\end{array}$ & $\begin{array}{c}\text { Obs-calc } \\
(\mathrm{MHz})\end{array}$ \\
\hline 44 & 21 & 24 & 44 & 19 & 25 & $v_{0}$ & $\begin{array}{c}0.630 \mathrm{E}-03 \\
0.300 \mathrm{E}-04 \\
0.420 \mathrm{E}-04 \\
0.750 \mathrm{E}-04 \\
0.690 \mathrm{E}-04 \\
0.120 \mathrm{E}-04 \\
0.500 \mathrm{E}-04 \\
0.140 \mathrm{E}-04\end{array}$ & $\begin{array}{l}303799.500 \\
303807.188 \\
303850.906 \\
303896.844 \\
303920.562 \\
303934.562 \\
303945.344 \\
303956.312\end{array}$ & 0.145 \\
\hline 24 & 1 & 24 & 23 & 1 & 23 & $\nu_{7}$ & $\begin{array}{l}0.850 \mathrm{E}-03 \\
0.440 \mathrm{E}-04 \\
0.120 \mathrm{E}-04\end{array}$ & $\begin{array}{l}303985.531 \\
304004.344 \\
304017.406\end{array}$ & 0.158 \\
\hline 51 & 27 & 24 & 51 & 27 & 25 & $\nu_{7}$ & $\begin{array}{l}0.150 \mathrm{E}-04 \\
0.110 \mathrm{E}-04 \\
0.760 \mathrm{E}-04 \\
0.950 \mathrm{E}-05 \\
0.720 \mathrm{E}-04\end{array}$ & $\begin{array}{l}304041.969 \\
304082.000 \\
304083.469 \\
304084.906 \\
304104.406\end{array}$ & 0.233 \\
\hline 43 & 20 & 24 & 43 & 19 & 25 & $v_{0}$ & $\begin{array}{l}0.720 \mathrm{E}-03 \\
0.130 \mathrm{E}-04 \\
0.440 \mathrm{E}-04 \\
0.110 \mathrm{E}-04 \\
0.370 \mathrm{E}-04\end{array}$ & $\begin{array}{l}304109.156 \\
304122.500 \\
304132.969 \\
304143.469 \\
304147.594\end{array}$ & 0.028 \\
\hline 14 & 12 & 2 & 13 & 12 & 1 & $\nu_{7}$ & $\begin{array}{l}0.710 \mathrm{E}-04 \\
0.120 \mathrm{E}-04 \\
0.120 \mathrm{E}-04\end{array}$ & $\begin{array}{l}304183.906 \\
304205.594 \\
304230.000\end{array}$ & -0.087 \\
\hline 23 & 2 & 22 & 22 & 2 & 21 & $\nu_{7}$ & $\begin{array}{c}0.690 \mathrm{E}-03 \\
0.700 \mathrm{E}-05 \\
0.700 \mathrm{E}-05 \\
0.660 \mathrm{E}-04 \\
0.340 \mathrm{E}-04 \\
0.300 \mathrm{E}-04 \\
0.420 \mathrm{E}-04 \\
0.860 \mathrm{E}-05 \\
0.100 \mathrm{E}-04 \\
0.860 \mathrm{E}-05 \\
0.690 \mathrm{E}-05 \\
0.310 \mathrm{E}-04 \\
0.170 \mathrm{E}-04\end{array}$ & $\begin{array}{l}304260.281 \\
304265.406 \\
304274.094 \\
304277.125 \\
304281.281 \\
304314.219 \\
304325.156 \\
304335.406 \\
304343.094 \\
304346.688 \\
304356.688 \\
304367.094 \\
304383.938\end{array}$ & -0.031 \\
\hline 42 & 19 & 24 & 42 & 18 & 25 & $v_{0}$ & $\begin{array}{l}0.820 \mathrm{E}-03 \\
0.260 \mathrm{E}-04 \\
0.120 \mathrm{E}-04 \\
0.790 \mathrm{E}-05 \\
0.770 \mathrm{E}-04 \\
0.640 \mathrm{E}-04\end{array}$ & $\begin{array}{l}304402.219 \\
304405.969 \\
304415.438 \\
304420.688 \\
304422.344 \\
304439.188\end{array}$ & -0.083 \\
\hline 42 & 37 & 6 & 42 & 35 & 7 & $v_{0}$ & $\begin{array}{l}0.140 \mathrm{E}-04 \\
0.840 \mathrm{E}-05\end{array}$ & $\begin{array}{l}304459.594 \\
304463.469\end{array}$ & -0.095 \\
\hline 23 & 15 & 9 & 22 & 17 & 6 & $v_{0}$ & $0.110 \mathrm{E}-04$ & 304482.562 & -0.212 \\
\hline
\end{tabular}

tion used between the known lines. Over most of the BWO range, this absolute absorption calibration is good to $\sim 10 \%$. However, there exist narrow regions separated by $\sim 10 \mathrm{GHz}$ where the frequency and amplitude variations of the BWO are much steeper functions of the voltage. In these regions, uncertainties of $\geqslant 100 \%$ are possible. Clearly, in all regions the uncertainty in the absolute coefficient is directly related to the distance in frequency to the nearest calibration point.

The final product of this is a line list of observed transitions, an example of which is shown in Table I for a small portion of the spectrum shown in Fig. 5.

To measure linewidths and calculate pressure broadening parameters, the spectrum must be recorded in the true line shape mode as shown in Fig. 8. Initially the spectrum is analyzed for center frequency and amplitude information as described above. Then:

(8) Selection criteria based on line strength and spectral congestion are used to select lines for line shape analysis.

(9) Voigt profiles, with additional linear terms to allow for base line slope, are fit to the original data files via nonlinear least squares methods and these measured linewidths added to the line list.

(10) For the measurement of pressure broadening parameters, data at several pressures which result in linewidths which vary from $\sim 1.5$ Doppler widths to $\sim 15$ Doppler widths are recorded and the software correlates the results from the several pressures and preforms fits to determine the pressure broadening parameters for each line. 
TABLE II. Rotational constants for the $\nu_{7}$ vibrational state of $\mathrm{HNO}_{3}(\mathrm{MHz})$.

\begin{tabular}{ccc}
\hline \hline Parameter & & Value $(\sigma)$ \\
\hline$A$ & & $13028.9751(7)$ \\
$B$ & & $12098.5690(6)$ \\
$C$ & $\times 10^{1}$ & $6201.616(8)$ \\
$\Delta_{J}$ & $\times 10^{1}$ & $0.145517(9)$ \\
$\Delta_{J K}$ & $\times 10^{2}$ & $-0.200608(31)$ \\
$\Delta_{K}$ & $\times 10^{2}$ & $0.601531(256)$ \\
$\delta_{J}$ & $\times 10^{1}$ & $0.12814(9)$ \\
$\delta_{K}$ & $\times 10^{7}$ & $-0.27861(16)$ \\
$H_{J}$ & $\times 10^{6}$ & $0.3522(93)$ \\
$H_{J K}$ & $\times 10^{6}$ & $-0.43719(641)$ \\
$H_{K J}$ & $\times 10^{6}$ & $0.985530(7125)$ \\
$H_{K}$ & $\times 10^{7}$ & $-0.62083(178)$ \\
$h_{J}$ & $\times 10^{6}$ & $-0.133(5)$ \\
$h_{J K}$ & $\times 10^{11}$ & $0.1705(316)$ \\
$L_{J K}$ & $\times 10^{11}$ & $-0.210(42)$ \\
$L_{J K}$ & & $0.397(55)$ \\
\hline \hline
\end{tabular}

\section{SPECTROSCOPIC RESULTS}

\section{A. The analysis of the rotational structure of the $\nu_{7}$ vibrational state of $\mathrm{HNO}_{3}$}

As a specific example, consider the spectroscopy of the rotational structure of the $\nu_{7}$ vibrational state of nitric acid $\left(\mathrm{HNO}_{3}\right)$. Nitric acid has been studied extensively in the $\mathrm{mm} /$ submm and the infrared because of its fundamental role in chemistry and because it is an important component in the ozone destruction cycle in the upper atmosphere. We have previously reported an analysis of the $\nu_{7}$ state which used a phase locked, harmonic generation system. ${ }^{40}$ In that study, about 140 rotational lines in the $100-600 \mathrm{GHz}$ region were identified and fit to a Watson Hamiltonian ${ }^{41}$ with a rms deviation of $0.068 \mathrm{MHz}$.

With the new FASSST system, 510 lines were identified and measured in the $247-375 \mathrm{GHz}$ region. These were combined with the earlier lines in a Watson analysis with an overall rms deviation of $0.081 \mathrm{kHz}$. Table II shows the spectral constants which result form this fit, and Table I includes examples of that fit to the $\nu_{7}$ experimental data set. For the observed amplitudes, there are no adjustable parameters because in rotational spectra it is possible to calculate absolute absorption coefficients from the known dipole moment and rotational constants.

All of the 510 lines were acquired (along with many thousands of lines in other vibrational states) in a total scan time of $12.5 \mathrm{~s}$, and their frequencies automatically calculated via the procedures discussed above. For these measurements, averages of either two or three separate scans were used. Because the data acquisition time per scan is so short, it would be practical to average a much larger number of scans to further improve the measurement accuracy. In Fig. 6 above a number of these $\nu_{7}$ lines have been indicated with a star.

\section{B. The pressure broadening of $\mathrm{HNO}_{3}$}

In addition to line frequencies and amplitudes, linewidths are important spectral parameters. Because it is easiest to maximize dynamic range by insertion of a low fre-
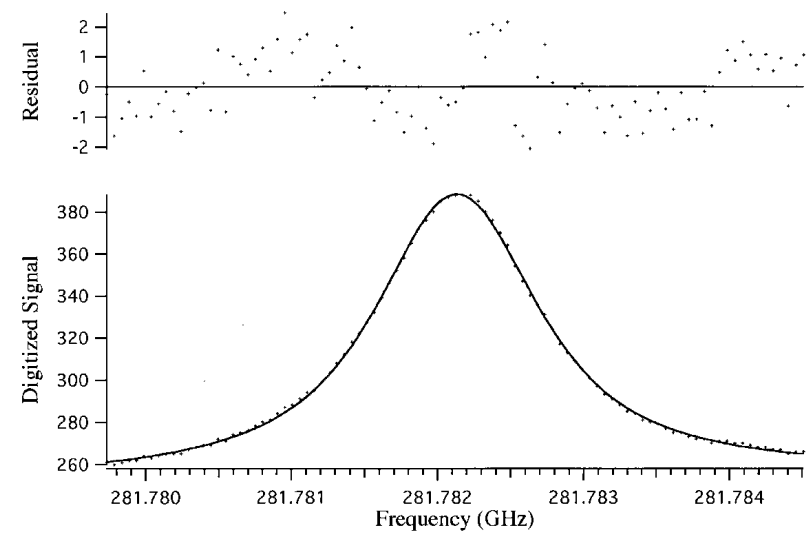

FIG. 9. A Voigt fit to an observed transition of $\mathrm{HNO}_{3}$.

quency roll off between the $\mathrm{mm} / \mathrm{submm}$ detector and the $\mathrm{A} / \mathrm{D}$ input to the computer, the spectral line shape is distorted in experiments designed to optimize $\mathrm{S} / \mathrm{N}$. However, as shown above in Fig. 7, the dynamic range problem is not severe, and it is possible to select bandwidths which preserve line shape. This true line shape regime is the one in which we ordinarily make pressure broadening measurements and which we have described in detail elsewhere. ${ }^{6}$

For the pressure broadening measurements reported here, nonlinear Voigt line fitting procedures were used to calculate the Lorentzian half-width. Figure 9 shows the results of such a fit. Software then collates the results of spectral scans made at $\sim 15$ different pressures and least squares procedures are used to calculate the pressure broadening coefficient of each line. Figure 10 shows a plot of the Lorentzian half-width recovered from such fits as a function of pressure.

Using conventional phase lock techniques, we have previously studied the pressure broadening of 16 lines of $\mathrm{HNO}_{3} .{ }^{42}$ Four of these overlap the current work and a comparison is shown in Table III. Inspection of Table III shows that the average difference is $\sim 3 \%$, well within the $5 \%-$ $10 \%$ uncertainty typically reported for pressure broadening measurements.

\section{DISCUSSION AND CONCLUSIONS}

In this article, we have discussed a new kind of $\mathrm{mm} / \mathrm{sub}$ $\mathrm{mm}$ spectrometer of unique and powerful capabilities. By combining a fast scanning technology and optical calibration

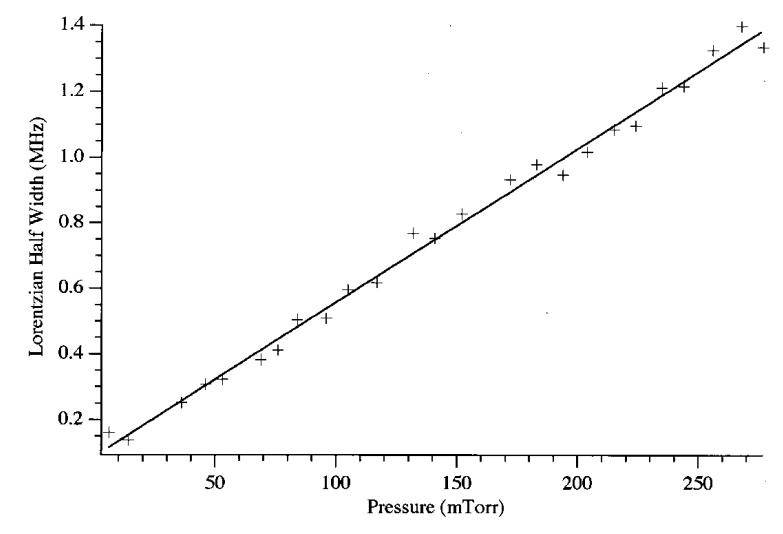

FIG. 10. The Lorentzian half-width recovered from the Voigt fits as a function of pressure. 
TABLE III. A comparison between pressure broadening parameters of $\mathrm{HNO}_{3}$ measured using conventional phase lock techniques and those obtained with the FASSST system.

\begin{tabular}{ccccc}
\hline \hline $\begin{array}{c}\text { Transition } \\
J_{K_{a}^{\prime} K_{c}^{\prime}}^{\prime}-J_{K_{a}^{\prime \prime} K_{c}^{\prime \prime}}^{\prime \prime}\end{array}$ & $\begin{array}{c}\text { Frequency } \\
(\mathrm{MHz})\end{array}$ & $\begin{array}{c}\gamma\left(\mathrm{N}_{2}\right) \\
(\mathrm{MHz} / \mathrm{Torr})\end{array}$ & $\begin{array}{c}\gamma^{a}\left(\mathrm{~N}_{2}\right) \\
(\mathrm{MHz} / \mathrm{Torr})\end{array}$ & $\begin{array}{c}\% \\
\text { difference }\end{array}$ \\
\hline $22_{2,22}-21_{0,21}$ & 281685.373 & $4.42 \pm 0.06$ & $4.19 \pm 0.04$ & 5.3 \\
$19_{3,16}-18_{3,15}$ & 281782.006 & $4.68 \pm 0.08$ & $4.59 \pm 0.03$ & 2.0 \\
$14_{8,6}-13_{8,5}$ & 283003.022 & $4.67 \pm 0.14$ & $4.88 \pm 0.10$ & 4.3 \\
$13_{11,2}-12_{11,1}$ & 284871.069 & $4.90 \pm 0.41$ & $4.87 \pm 0.16$ & 0.6 \\
\hline \hline
\end{tabular}

${ }^{a}$ Results from Goyette et al. (Ref. 41).

methodologies, it has been possible to achieve the sensitivity and measurement accuracy associated with phase lock methods. Equally importantly, the instantaneous bandwidth of the BWO is retained and a simple system, capable of very rapid spectral analysis results.

None the less a number of enhancements are still possible. These include:

(1) The development of a more compact FP reference system: While in our laboratory system, the folded $38.89 \mathrm{~m}$ FP cavity is not inconvenient, in many applications, it would be a limiting factor. There are at least three approaches to reducing the size. First, the linear interpolation used to calculate frequencies can be replaced by an analysis which uses the local information about the FP mode spacing to determine the characteristics of the nonlinearities in the BWO frequency-voltage function. Second, because the nature of the nonlinearity is constant from sweep to sweep, methodologies which are based on translation of the cavity mirrors by small increments during the data acquisition would also provide a means for analysis of the nonlinearity. Since the current linear method introduces little error, it would appear that either of these approaches would lead to a reduction of at least a factor of 5 in the required cavity length. Finally, a White cell approach could be used to fold the long path length into $\sim 1 \mathrm{~m}$.

(2) Higher measurement accuracy: Saturation spectroscopy makes possible higher measurement accuracy than ordinarily used spectroscopically. A very nice recent example has used phase locked BWOs to measure Lamb dip and crossover phenomena in ammonia $\left(\mathrm{NH}_{3}\right){ }^{43}$ We would expect that this and a combination of the approaches considered in (1) would result in increasing the measurement accuracy of the FASSST system by about another order of magnitude.

(3) Improved amplitude calibration: Amplitude calibration is achieved by linear interpolation between spectral lines of known intensity. Over most of the BWOs sweep range, this procedure is good to perhaps $10 \%$-a useful accuracy for most applications. However, over small regions the BWO power varies rapidly by $\pm x 2$ and independent means are required, especially if there are no nearby reference lines. Because these amplitude variations are fundamental properties of the BWO and are stable in time, absolute calibration can be achieved by recording a chopped sweep alternatively with a spectral scan.

Finally we should note that a much simplified version of this high resolution spectrometer should be a very attractive analytical gas analyzer whose capabilities are complimentary to the widely use FTIR gas analyzer. Such a system would be absolutely specific (because of the high spectral resolution), even for complex mixes of gases; very fast (in most applications, a full band sweep is not required and the total data acquisition time could be $\ll 1 \mathrm{~s}$ ); very sensitive (especially for small samples at low pressure), and very compact (for identification rather than spectral analysis, the FP requirements are significantly relaxed).

\section{ACKNOWLEDGMENTS}

The authors would like to thank the Air Force Office of Scientific Research for an equipment grant which made this work possible, and the Army Research Office and NASA for the support of our work. F.C.D. would also like to thank the Max Planck Society and the Alexander von Humboldt Foundation for a Max Planck Prize, whose proceeds also contributed to this work.

${ }^{1}$ W. C. King and W. Gordy, Phys. Rev. 90, 319 (1953).

${ }^{2}$ C. A. Burrus, Jr. and W. Gordy, Phys. Rev. 92, 274 (1953).

${ }^{3}$ W. C. King and W. Gordy, Phys. Rev. 93, 407 (1954).

${ }^{4}$ H. M. Pickett, Appl. Opt. 19, 2745 (1980).

${ }^{5}$ A. Bauer, M. Godon, M. Kheddar, J. H. Hartmann, J. Bonamy, and D. Robert, J. Quant. Spectrosc. Radiat. Transf. 37, 531 (1987).

${ }^{6}$ T. M. Goyette, F. C. De Lucia, J. M. Dutta, and C. R. Jones, J. Quant. Spectrosc. Radiat. Transf. 49, 485 (1993).

${ }^{7}$ J. K. Messer and F. C. De Lucia, Phys. Rev. Lett. 53, 2555 (1984).

${ }^{8}$ J. C. Pearson, L. C. Oesterling, E. Herbst, and F. C. De Lucia, Phys. Rev. Lett. 75, 2940 (1995).

${ }^{9}$ F. C. De Lucia, Appl. Phys. Lett. 31, 606 (1977).

${ }^{10}$ D. D. Skatrud and F. C. De Lucia, Appl. Phys. Lett. 46, 631 (1985).

${ }^{11}$ H. O. Everitt, D. D. Skatrud, and F. C. De Lucia, Appl. Phys. Lett. 49, 995 (1986).

${ }^{12}$ K. V. Chance, D. G. Johnson, W. A. Traub, and K. W. Jucks, Geophys. Res. Lett. 18, 1003 (1991).

${ }^{13}$ J. W. Waters, J. C. Hardy, R. F. Jarnot, and H. M. Pickett, Science 214, 61 (1981).

${ }^{14}$ B. Carli and J. H. Park, J. Geophys. Res. 93, 3851 (1988).

${ }^{15}$ E. Herbst, Annu. Rev. Phys. Chem. 46, 27 (1995).

${ }^{16}$ T. G. Phillips, in The Physics and Chemistry of Interstellar Molecular Clouds, edited by G. Winnewisser and G. C. Pelz (Springer, New York, 1995), p. 344.

${ }^{17}$ K. Menten and K. Young, Astrophys. J. L67, 450 (1995).

${ }^{18}$ E. C. Sutton, R. Peng, W. C. Danchi, P. A. Jaminet, G. Sandell, and A. P. G. Russell, Astrophys. J. Supp. 97, 455 (1995).

${ }^{19}$ P. Helminger, F. C. De Lucia, and W. Gordy, Phys. Rev. Lett. 25, 1397 (1970).

${ }^{20}$ P. Helminger, J. K. Messer, and F. C. De Lucia, Appl. Phys. Lett. 42, 309 (1983).

${ }^{21}$ S. S. Sussman, B. C. Johnson, J. M. Yarborough, H. E. Puthoff, R. H. Pantell, and J. SooHoo, in Submillimeter Waves, edited by J. Fox (Polytechnic, New York, 1971), p. 211.

${ }^{22}$ K. M. Evenson, D. A. Jennings, and F. R. Peterson, Appl. Phys. Lett. 44, 576 (1984). 
${ }^{23}$ E. R. Brown, K. A. McIntosh, K. B. Nichols, and C. L. Dennis, Appl. Phys. Lett. 66, 285 (1995).

${ }^{24}$ D. D. Bicanic, B. F. J. Zuidberg, and A. Dymanus, Appl. Phys. Lett. 32, 367 (1978).

${ }^{25}$ W. A. M. Blumberg, H. R. Fetterman, D. D. Peck, and P. F. Goldsmith, Appl. Phys. Lett. 35, 582 (1979).

${ }^{26}$ M. Ino, T. Ishibashi, and M. Ohmori, Jpn. J. Appl. Phys. Suppl. 16-1, 898 (1977).

${ }^{27}$ E. R. Brown, J. R. Soderstrom, C. D. Parker, L. J. Mahoney, K. M. Molvar, and T. C. McGill, Appl. Phys. Lett. 58, 2291 (1991).

${ }^{28}$ R. Kompfner and N. T. Williams, Proc. IRE 41, 1602 (1953).

${ }^{29}$ T. Yeou, in: Proceedings of the 5th International Congress on Microwave Tubes, Paris, 1964 (Academic, New York, 1965).

${ }^{30}$ M. B. Golant, R. L. Vilenskaya, E. A. Zyulina, Z. F. Kaplun, A. A. Negirev, V. A. Parilov, T. B. Rebrova, and V. S. Savelev, Prib. Tekh. Eksp. 4, 136 (1964).

${ }^{31}$ M. B. Golant, Z. T. Alekseenko, Z. S. Korotkova, L. A. Lunkina, A. A. Negirev, O. P. Petrova, T. B. Rebrova, and V. S. Savelev, Prib. Tekh. Eksp. 3, 231 (1969).

${ }^{32}$ A. F. Krupnov, L. I. Gershtein, V. G. Shustrov, and S. P. Belov, Izv. VUZ. Radiofizika 13, 1403 (1970).
${ }^{33}$ Z. Kisiel, Chem. Phys. Lett. 118, 334 (1985).

${ }^{34}$ A. F. Krupnov and A. V. Burenin, in Molecular Spectroscopy: Modern Research, edited by K. N. Rao (Academic, New York, 1976), Vol. II.

${ }^{35}$ G. Winnewisser, A. F. Krupnov, M. Yu. Tretyakov, M. Liedtke, F. Lewen, A. H. Saleck, R. Schieder, A. P. Shkaev, and S. V. Volokov, J. Mol. Spectrosc. 165, 294 (1994).

${ }^{36}$ S. P. Belov, F. Lewen, Th. Klaus, and G. Winnewisser, J. Mol. Spectrosc. 174, 606 (1995).

${ }^{37}$ Y. A. Dryagin, Izv. VUZ. Radiofizika 13, 141 (1970).

${ }^{38}$ P. N. Robson, in Spectroscopic Techniques, edited by D. H. Martin (Elsevier, New York, 1967).

${ }^{39}$ The detailed circuit diagrams and descriptions are available from F.C.D. upon request.

${ }^{40}$ R. A. Booker, R. L. Crownover, and F. C. De Lucia, J. Mol. Spectrosc. 128, 62 (1988).

${ }^{41}$ J. K. G. Watson, J. Chem. Phys. 45, 1360 (1966).

${ }^{42}$ T. M. Goyette, W. L. Ebenstein, and F. C. De Lucia, J. Mol. Spectrosc. 128, 108 (1988).

${ }^{43}$ S. P. Belov, Th. Klaus, G. M. Plummer, R. Schieder, and G. Winnewisser, Z. Naturforsch. Teil A 50, 1187 (1995). 